\title{
Chemical Education
}

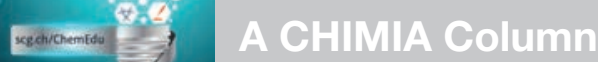

\section{Interdisciplinary Projects Applied to Chemistry Lessons: Example of Chemistry Linked to Food Science}

\section{Lydie Moreau*a and Véronique Breguet Mercier ${ }^{\star b}$}

${ }^{\star}$ Correspondence: Prof. L. Moreaua, E-mail: Lydie.Moreau@hevs.ch; Dr. V. Breguet

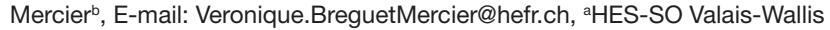
(Institut Technologie du Vivant), ' HEIA Fribourg (Institut ChemTech)

Abstract: An interdisciplinary project regarding the effect of ascorbic acid on bread dough's physico-chemical properties was proposed to bachelor students in chemistry and food sciences. Such an approach was proposed to develop both scientific and soft skills, in order to prepare students for their future working environment. Together, students deepened their knowledge regarding food science and chemistry. They were then able to plan and design experiments demonstrating the impact of gluten network formation and ascorbic acid influence onto bread dough and finished product characteristics. This way of teaching was very appreciated by students, nevertheless it highlighted the fact that the professors' investment was considerably high, and that good organization, alignment and preparation prior to the start of this project is key.

Keywords: Chemical education · Food science · Interdisciplinarity · Project organisation

Universities of Applied Sciences, such as HES-SO, are aiming at preparing future engineers to work in technical fields mainly in the private sector. Companies, regardless of their size and activities, are working through projects that gather professionals from different disciplines. Therefore, students in chemistry will have to collaborate with non-chemists as soon as they leave school.

Chemistry enables to identify and follow-up reactions during the process-making steps of several products, including food products. During the bread-making process, a series of chemical reactions were identified such as those leading to and/or modifying the gluten network. Teaching specific chemical processes through examples of commonly used products, such as bread, can help engage the students in the course.

In the context of their bachelor studies, an interdisciplinary project was set up between food science and chemistry students. The aim was 2-fold:

- Such projects allowed the students to be better prepared for their future jobs in industries, as project management experience (objective definition, planning, experimental design identification...), and some soft skills could be developed (communication, problem solving...)

- Chemistry students had the opportunity to search for information to understand the chemistry behind bread-dough formation process to discuss the trial observations (implying a high level of understanding), while food science students could deepen their knowledge regarding chemistry applied to food products.

\section{Chemistry Behind Bread-dough-making Process and Impact of Ascorbic Acid Addition}

During the kneading step (prior to the fermentation and cooking steps), gluten proteins from wheat will interact with each other, forming a network. Such a protein network is necessary for the product to keep its shape and retain the $\mathrm{CO}_{2}$ generated during the fermentation process (leading to dough leavening). Gluten proteins consist of polymeric glutenin and monomeric gliadin, each of them playing a specific role regarding dough rheology. ${ }^{[1]}$ Glutenin builds up the polymeric protein network that provides cohesiveness and elasticity to the dough, whereas gliadins contribute to dough viscosity and extensibility. Intermolecular disulfide (SS) bonds between glutenin polymers are key to the protein network. The oxidation of free thiol (SH) groups to SS bonds, which increases the molecular weight of the glutenin aggregates, as well as SH-SS exchange reactions, are important to build a three-dimensional gluten network during mixing. ${ }^{[1]}$

Redox agents are frequently used in the bread-making industry to optimize gluten performance. Ascorbic acid is used as a bread improver, as its addition to dough causes an increase in loaf volume and an improvement in crumb structure. ${ }^{[2]}$ It also leads to dough strengthening. ${ }^{[1]}$ Ascorbic acid is a reducing agent but it is converted to dehydroascorbic acid during the mixing stage in the presence of oxygen thanks to ascorbic acid oxidase (naturally present in wheat flour) (Scheme 1).

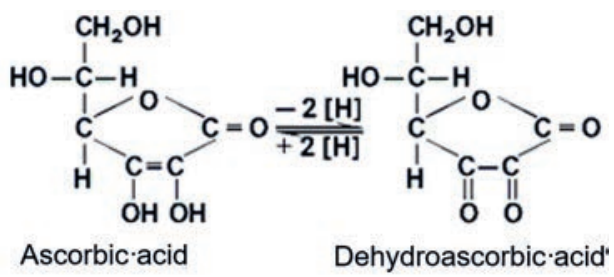

Scheme 1. Ascorbic acid conversion into dehydroascorbic acid in the presence of oxygen, catalyzed by an ascorbic acid oxidase endogenous to wheat flour. ${ }^{[3]}$

Dehydroascorbic acid will then interact with glutathione (which is a tri-peptide: glutamic acid-cysteine-glycine endogenous to wheat flour and can also come from yeast cells) to move from the reduced form GSH into the oxidized form GSSG (Scheme 2).
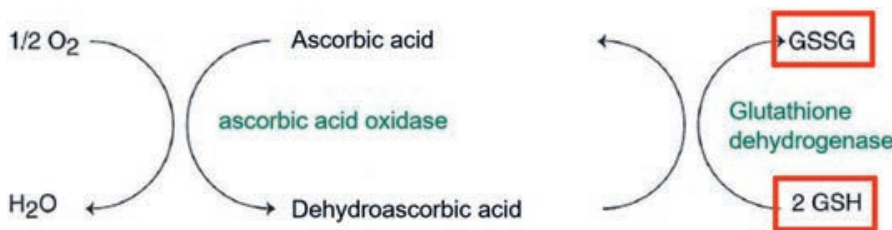

Scheme 2. Formation of oxidized glutathione (GSSG) via the action of dehydroascorbic acid and catalyzed by glutathione dehydrogenase. ${ }^{[3]}$ 
The subsequent interactions between GSSH and gluten proteins are unclear despite several research projects according to a systematic review performed in 2019. ${ }^{[1]}$ A few hypotheses were proposed. Among them, Grosch and colleagues ${ }^{[4-6]}$ proposed that, as GSH in dough is rapidly oxidized, it prevents GSH from cleaving specific disulphide bonds in gluten (producing poor dough properties). Kuninori and Nishiyama[7] suggested that GSSG promotes inter-protein disulphide bonds (P-SS-P') through a disulphide-thiol interchange reaction:

$$
\mathrm{GSSG}+\mathrm{P}-\mathrm{SH}+\mathrm{P}^{\prime}-\mathrm{SH} \rightarrow 2 \mathrm{GSH}+\mathrm{P}-\mathrm{SS}-\mathrm{P}^{\prime}
$$

However, according to Faccio et al. ${ }^{[8]}$ the presence of GSSG leads to possible weakening of thiol/disulfide-exchange reactions causing the slow depolymerization of gluten proteins by opening the disulfide bonds of the protein network (Scheme 3 ).
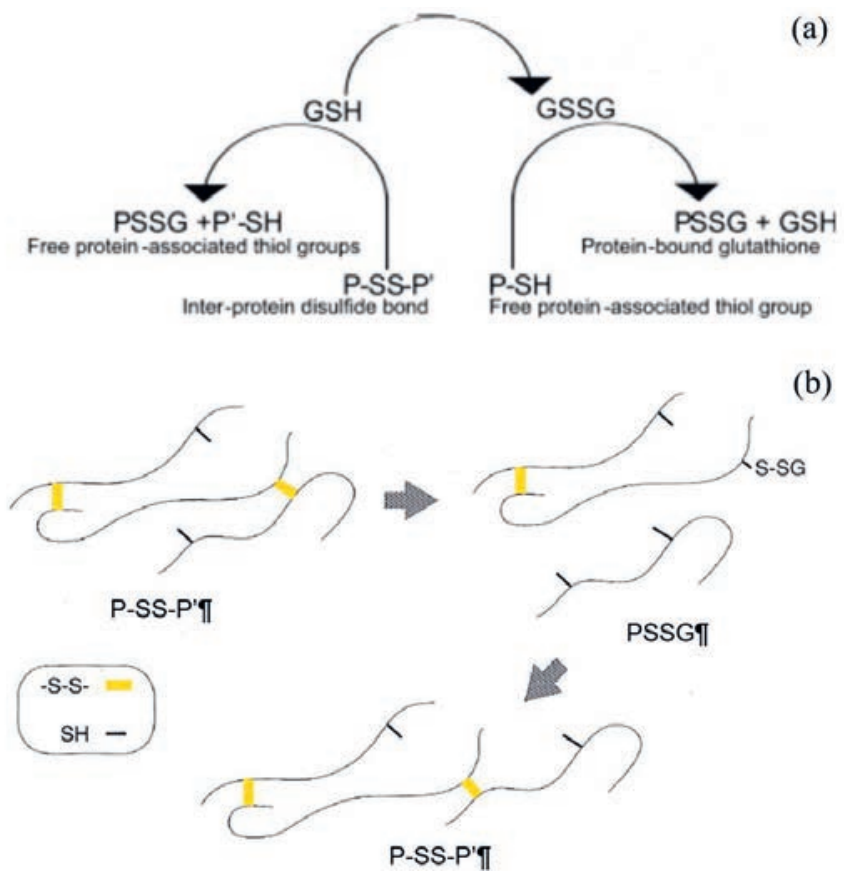

Scheme 3. (a) Interactions between glutathione (either oxidized or reduced) and proteins during bread dough kneading ${ }^{[9]}$ and (b) rupture and formation of disulfide bonds from gluten proteins during kneading step $^{[3]}$ : (PSSG: protein bound glutathione)

\section{Implementation of an Interdisciplinary Project Involving Chemistry and Food Science Students}

As chemistry reactions can explain physico-chemical properties of bread dough formation, an interdisciplinary project was set between students from two different fields of HES-SO (chemistry and food science). Student groups were formed with 2 to 5 persons from each discipline (depending on the year). They collaborated over a period of 3 months to study the impact of ascorbic acid on bread dough. The following practical objectives were defined and communicated to the students at the beginning of the project:

- Define the process and formulation parameters impacting the physico-chemical characteristics of bread (dough and finished product) with a focus on the role of ascorbic acid,

- Plan, conduct and interpret the trials (kitchen and pilot plant), taking into account the available literature,

- Write a report and orally present the results.

The following pedagogical objectives were also defined, and the project was organized accordingly:

- Exchange knowledge between food science students and chemistry students,

- Communicate efficiently within the team,
- Collaborate and share the tasks to reach the common practical objectives.

In terms of methods to make and characterize the dough and the finished product, very simple measurements were performed by the students and therefore could be implemented in most schools. For example, they used a graduated cylinder to assess dough volume changes during fermentation as well as a simple ruler to observe dough elasticity. Of course, more sophisticated methods can be used if the equipment is available.

As chemistry and food science students were not in the same location and had many agenda conflicts, the following project organization was set-up (Fig. 1).

The following pedagogical documents were created prior to the project start and were communicated to the students. Writing these documents is of great help to ensure teachers' alignment and time spent on them should not be neglected:

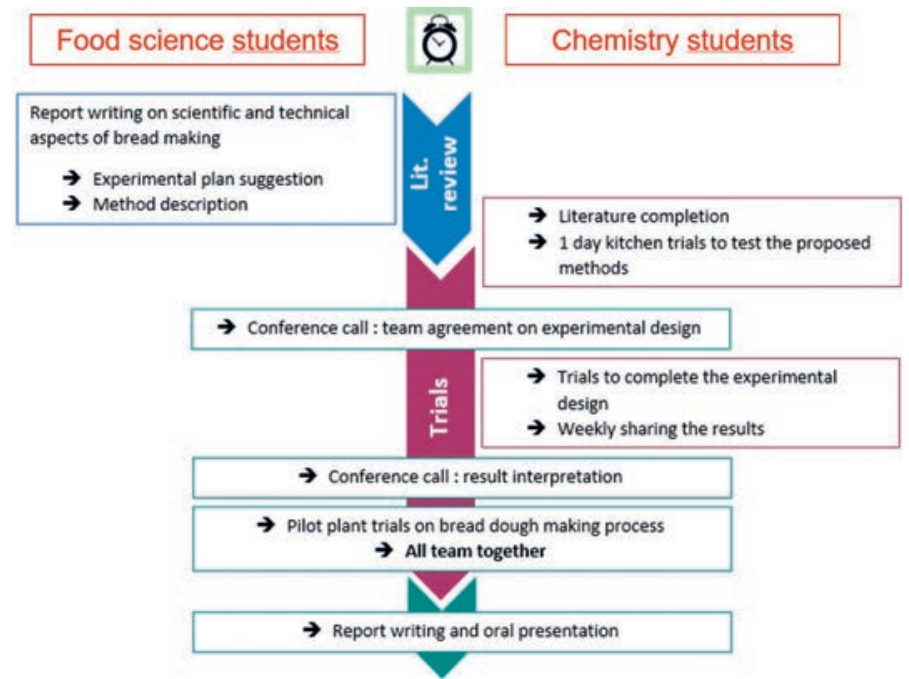

Fig. 1. Interdisciplinary project organization during a time frame of 3 months.

- Project rules: project objectives, milestones, roles and responsibilities, deliverables, evaluation.

- Evaluation modalities: common grids to evaluate the reports and the oral presentation made as a team. A separate evaluation was also performed for parts of the work which was done separately for each discipline.

- Feedback questionnaires to improve the project for the following year.

Main Outcomes from such an Interdisciplinary Project

This interdisciplinary project between students has several advantages. First, it is very appreciated by the students: feedback questionnaires filled out after completion of the project rated such initiatives with grades between 3 and 4 for $95 \%$ of the questions asked (18 questions in total, maximum grade being 4). Second, this school project allowed them to exchange key knowledge regarding the science behind bread making and how chemical reactions can have a significant impact onto food product characteristics (Scheme 2). It therefore enhanced their understanding of such mechanisms. Third, soft skills were developed such as communication as already reported in the literature. ${ }^{[10,11]}$

Working with students on two different geographical locations is a non-negligible parameter, which may affect success. Therefore, prior to the start of the project, the teacher should ensure that communication tools are known by the students. For example, online document sharing tools (Google ${ }^{\circledR}$ doc/slide/ sheet - Dropbox / SwitchDrive - Moodle) or online project management tools (such as Trello). 


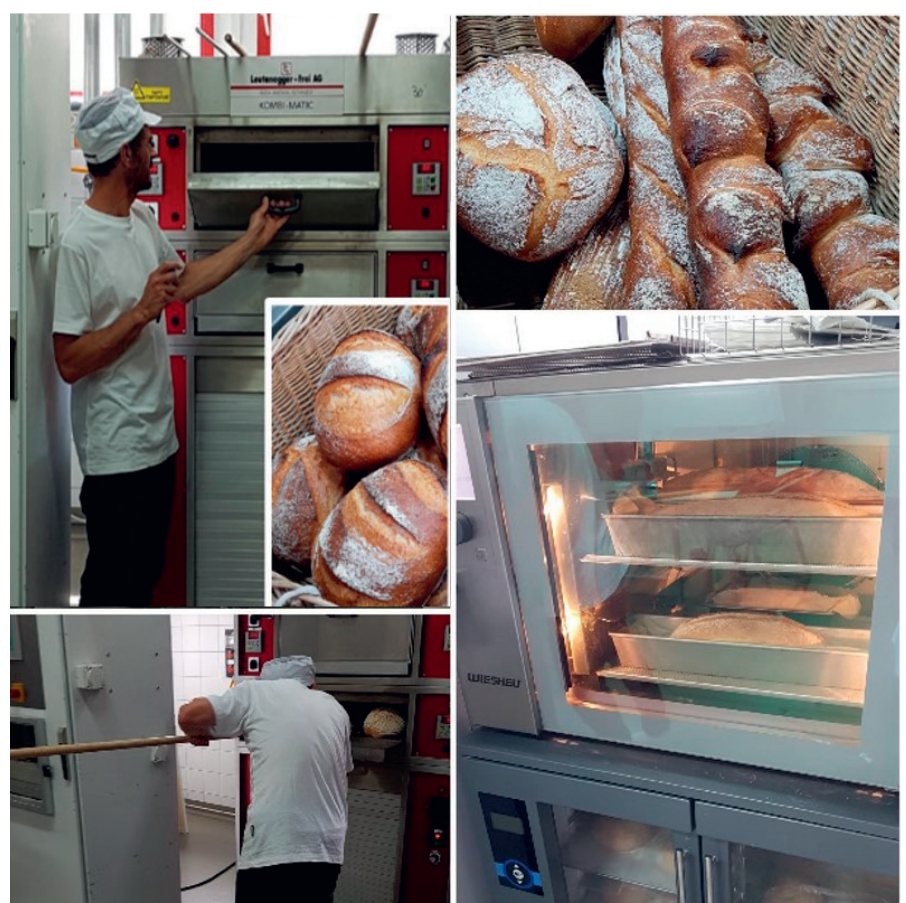

Fig. 2. Pilot scale trials performed at the end of the project to illustrate some observations made previously at laboratory scale.

Nevertheless, to ensure such interdisciplinary projects to be successful, they have to be very well prepared, which is time consuming; hence, the teacher's motivation is key. As it implies protagonists from different fields, with different skills and possibly from different geographic locations, this interdisciplinary work enhances possible misunderstanding issues. Consequently, the project has to be well-organized and follow-up is important. The workload asked to the students should not be underestimated as it might discourage some of them during the project. Teacher alignment prior to the start of the interdisciplinary project is also crucial.

Finally, it was observed that a special attention has to be paid to ECTS credits attributed to students: it should be well balanced between the fields for the student implication to be similar within a group.[12]

\section{Side note}

The first edition of this interdisciplinary project was organized in the context of a wider project on pedagogical innovation, looking at interdisciplinarity, and was financed by HES-SO

Received: December 12, 2019

[1] N. Ooms, J. A. Delcour, Curr. Opin. Food Sci. 2019, 25, 88

[2] W. Grosch, H. Wieser, J, Cereal Sci, 1999, 29, 1.

[3] P. Feillet, Institut National de la Recherche Agronomique, France, 2000.

[4] G. Mair, W. Grosch, J. Sci. Food Agricul. 1979, $30,914$.

[5] C. Walter, W. Grosch, J. Cereal Sci. 1987, 5, 299.

[6] R. Kieffer, J. J. Kim, C. Walther, G. Laskawy, W. Grosch, J. Cereal Sci. 1990, 11, 143.

[7] T. Kuninori, J. Nishiyama, Cereal Foods World 1993, 38, 554

[8] G. Faccio, L. Flander, J. Buchert, M. Saloheimo, E. Nordlund, J. Cereal Sci. 2012, 55, 37 .

[9] R. Sarwin, G. Laskawy, W. Grosch, Cereal Chem. 1993, 70, 553.

[10] D. Gachoud, S. Gallant, L. Lucarelli, N. Oberhauser, A. C. Allin-Pfister, Chêne Bourg, Suisse, 2017.

[11] P. Hall, J. Interprof. Care 2005, 19, 188.

[12] I. Oandasan, S. Reeves, J. Interprof. Care 2005, 19, 21. 